\title{
The impact of the theoretical properties of the Logistic Function on the generation of optically detectable watermarks
}

\author{
Aidan Mooney and John G. Keating \\ Department of Computer Science, N.U.I. Maynooth, Maynooth, \\ Co. Kildare, Ireland.
}

\begin{abstract}
A digital watermark is a visible, or preferably invisible, identification code that is permanently embedded in digital media, to prove owner authentication and provide protection for security or defence documents. In this paper, we present an approach for the generation of watermarks using a logistic chaotic function. Using this function, in conjunction with seed management, it is possible to generate chaotic sequences that may be used to create highpass or lowpass digital watermarks. A slight change in the initial conditions will quickly lead to a significant change in the subsequent states of the system, and thus will generate substantially different watermarks. This technique has been shown to offer an added security advantage over the more traditionally generated watermarks created from pseudorandom sequences, in that only the function seed needs to be stored. We have previously presented a study where an optical correlator was suitable for the detection of chaotically generated watermarks. We have also studied the impact of shot noise present in an optical detector for watermarks generated using the logistic function. The logistic function presented in this paper is ill-defined for certain seed values and has not been fully investigated for the purpose of watermark generation. We consider the impact of the theoretical properties of the logistic function on watermark generation and their highpass and lowpass properties, which when embedded in digital media, are suitable for optical detection.
\end{abstract}

Keywords: Digital Watermarking, Logistic Difference Equation, Optical Correlation, Lyapunov Exponent, Power Spectrum, Probability Density

\section{INTRODUCTION}

Digital Watermarking provides a means of inserting additional information into digital documents. This extra information may be used to provide a certain degree of protection of the document from malicious attack. This information is usually a piece of identifying information which may be used at a later date to prove ownership. The original document owner can prove ownership of copies by proving that it contains their original watermark.

Numerous watermark generation techniques have been proposed to date, ranging from the use of personal logos to the use of pseudorandom sequences of numbers ${ }^{1}$. The use of chaotic functions for the generation of watermarks has also been proposed by Pitas et al. ${ }^{2-4}$ where the Markov Maps and Bernoulli Maps are used as the chaotic functions. These types of watermark generation schemes require two values, the initial value and the function seed, in order to recreate the same watermark at a later stage. An advantage of these watermarks is their robustness to lowpass attacks. Typically these watermarks are distributed over the full cover image using an additive or multiplicative technique.

Pitas et al. have described in detail the use of chaotic functions in watermark generation ${ }^{2,4}$ and provide analysis of the functions and techniques used. A similar analysis for the logistic map does not exist and we believe that an understanding of it would be significant in the generation of chaotically generated watermarks.

The main difference between optical and digital watermarks lies in the retrieval process. Optical watermarking makes use of optical devices, like a photocopier, to retrieve the watermark, while digital watermarking uses computer software. To date, some optical watermarking approaches have been proposed, for example, Sun et

Further author information:

E-mail: amooney@cs.may.ie, jkeating@cs.may.ie 
al. presented an optical extraction technique which used an optical and visual means, like a photocopier and no digitization was required. ${ }^{5}$ Trustcopy have also introduced an optical watermarking technique but it uses paper and printers for verification of the watermark. Herrigel et al. present an optical/digital watermark identification system in which a reference number is extracted from the watermarked document using a lighting unit and optical component and compared with the original watermark. ${ }^{6}$ These techniques use optical techniques for the extraction of an embedded watermark but do not consider the use of optical techniques in watermark detection. We have examined chaos-based watermarks suitable for optical detection ${ }^{7,8}$ and refer to these as "optically detectable watermarks".

\section{FEATURES OF LOGISTIC EQUATION}

In this paper, we utilise watermarks that are generated using a chaotic function which is a function which is sensitive to initial conditions, is unpredictable, indecomposable and yet contains regularity. ${ }^{9}$ One motivation for using such chaotic functions to generate a watermark is that a single variable, $a$, seeding the chaotic function, will always result in the same output (mapping) when certain constraints or initial conditions are placed on the mapping. The primary advantage, however, is that it is possible to investigate the lowpass properties of the resulting watermark. A chaotic map is derived from a chaotic sequence fully described by the map $\left\{y_{j}: y_{j}=f\left(j_{j-1}, a\right)\right\}$ and an initial condition $y_{0}$. The function used in this paper for the generation of sequences which are transformed into watermarks is the logistic difference equation, sometimes referred to as the logistic equation or mapping:

$$
y_{n+1}=a y_{n}\left(1-y_{n}\right)
$$

where $a$ is the 'function seed' and $y_{n}$ is the current value of the mapping in time. ${ }^{10}$ The logistic difference equation was originally proposed to describe the dynamics of a population of insects in discrete generations. The value $y_{n+1}$ is dependant on its current density $y_{n}$. For low values of $a, y_{n}$ eventually converges to a single number as $n \rightarrow \infty$. When $a=3.0, y_{n}$ no longer converges - it oscillates between two values. This characteristic change in behaviour is called a bifurcation, as shown in Fig. 1(a). Increasing the value of $a(>3.0)$ causes $y_{n}$ to oscillate between not two, but four values. As $a$ increases, $y_{n}$ goes through bifurcations of period $2^{n}$ and eventually becomes chaotic. When the value of $a \approx 3.55699, y_{n}$ neither converges or oscillates - its value becomes completely random. For values of $a>3.5699$, the behaviour is largely chaotic ${ }^{10}$.
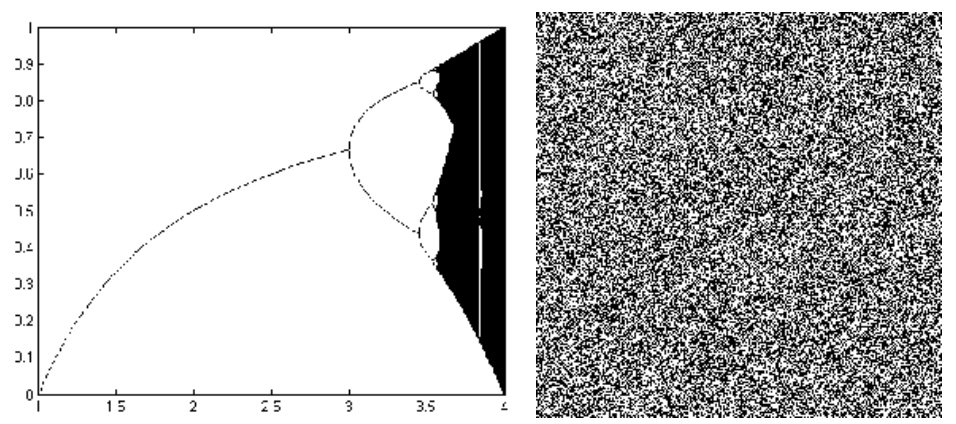

Figure 1. (a) Bifurcation Diagram for the Logistic Map (b) Watermark generated by iterating the Logistic Map with an initial value of 0.001 and a value of $a=4$.

This characteristic of the logistic map can be seen from the variation of the Lyapunov Exponent for the map with respect to the value of $a$. We can calculate the Lyapunov Exponent, $\lambda$, for the logistic equation by first performing the required differentiation 


$$
\begin{aligned}
y_{n+1} & =a y_{n}\left(1-x_{n}\right) \\
& =a y_{n}-a y_{n}^{2} \\
\frac{d y_{n+1}}{d y_{n}} & =a y_{n}^{0}-2 a y_{n}^{1} \\
& =a\left(1-2 y_{n}\right)
\end{aligned}
$$

which leads to the Lyapunov Exponent for the logistic equation

$$
\lambda=\lim _{N \rightarrow \infty} \frac{1}{N} \sum_{n=1}^{N} \log _{2}\left|a\left(1-2 y_{n}\right)\right|
$$

and is shown in Fig. 2. The Lyapunov Exponent is useful for distinguishing between the various types of orbits. When $\lambda \leq 0$ the logistic map exhibits periodic behaviour, and when $\lambda>0$ the logistic map exhibits chaotic behaviour ${ }^{11}, 12$. Fig. 2 illustrates the Lyapunov Exponent for the range $[0,4]$ for the logistic equation.

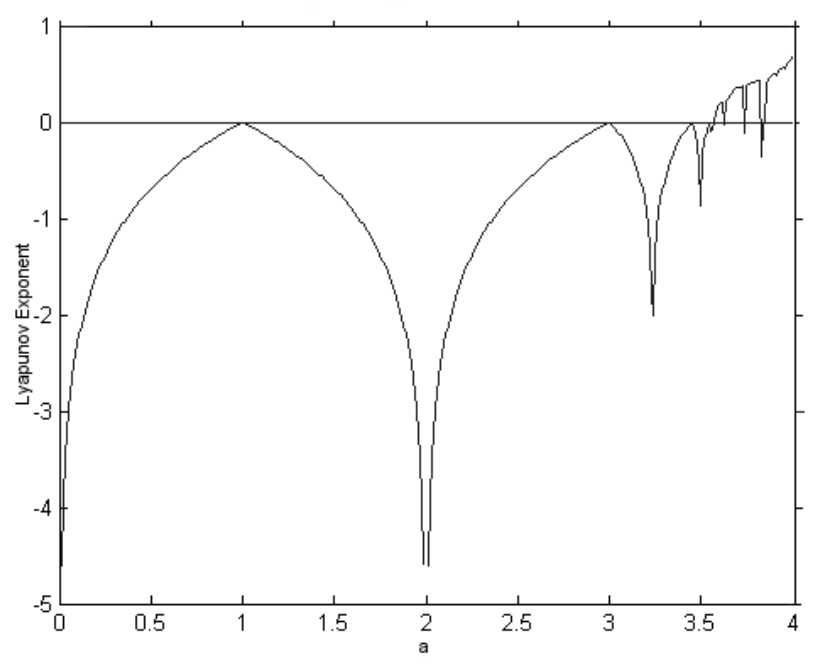

Figure 2. The Lyapunov Exponents for the Logistic Map.

Fig. 2 shows that for values of $a \lesssim 3.5699$ the value of the corresponding Lyapunov Exponent is negative and the logistic map exhibits periodic behaviour in this range. For values of $a \gtrsim 3.599$ the value of corresponding Lyapunov Exponent is positive and the logistic map exhibits chaotic behaviour in this range. When $a=2$ the value attained for the Lyapunov Exponent is $-\infty$ and this represents a superstable fixed point.

\section{WATERMARK GENERATION}

When the logistic equation is seeded with a value $3.5699 \leq a \leq 4.0$ (the chaotic region), and iterated, chaotic behaviour is witnessed. It is this feature of the logistic equation that we utilise to generate watermarks. The trajectorys produced for maps of this equation will differ greatly even for small differences in the seed value for the equation, provided the seed is within the chaotic region.

The 1D sequence generated by iterating this map is a sequence of real numbers between 0 and 1 and are converted to one of two values:

$$
y_{n}= \begin{cases}0 & y_{n} \leq 0.5 \\ 1 & y_{n}>0.5\end{cases}
$$


where 0.5 is a threshold and $y_{n}$ is the $n^{\text {th }}$ value in the sequence generated by the logistic equation.

This sequence (a series of zeros and ones) is then converted into a $2 \mathrm{D}$ image which may be used as a watermark. In this paper, we generate watermarks that are the same size as the image which will contain the watermark. A scanning technique, known as Peano Scanning, is used to determine watermark pixel ordering and is preferable to the conventional Raster Scanning technique in that scanning is not predictable, and can produce many variations within the same image. A drawback with using Peano Scanning is that it will only operate on images whose height and width are multiples of two. The scanning order always moves to a neighbouring pixel, but the pattern may appear in different orientations depending on the starting pixel within the scanning routine ${ }^{13,14}$. An example of a watermark generated using this technique is shown in Fig. 1(b). In this case the logistic function was seeded with a value $a=4$ and an initial starting value of $y_{0}=0.001$.

As the watermarks produced using chaotic function are reproducible, only the initial starting value and seed are needed to generate a sequence of numbers. This ensures that the sequence can only be reproduced if someone has the initial starting value and also the function seed. Given that the logistic map is not invertible, there is added security over pseudorandom sequences.

The logistic map maps the closed interval $[0,1]$ onto itself and examination of one such transformation (when $a=4$ ) illustrates this, as shown in Fig. 3 and may also be observed by examining Fig. 1 at $a=4$. By choosing an initial value $y_{0} \in[0,1]$ the successive states of the system are given by the trajectory ${ }^{20}$ :

$$
y_{0}, F\left(y_{0}\right), F^{2}\left(y_{0}\right)=F\left(F\left(y_{0}\right)\right), \ldots
$$

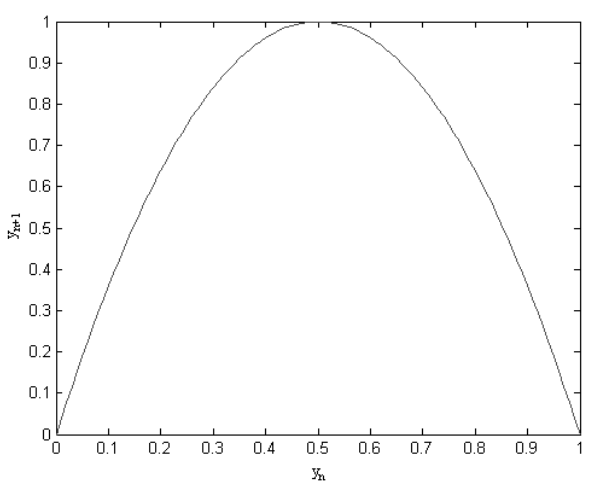

Figure 3. Transformation when $a=4$.

Fig. 4 shows the trajectories generated for two initial states. For both these cases the function seed, $a=4$ and the trajectories were plotted over 200 iterations. It can be seen that the trajectories are chaotic and also that with a slight change in the initial state, $10^{-3}$ in this case, the resultant trajectory is significantly altered. This property is known as the "Butterfly Effect" or more technically the "sensitive dependence on initial conditions".

\section{WATERMARK EMBEDDING AND DETECTION}

Watermark embedding is the process of inserting the generated watermark into some cover data. The generated watermark is embedded in the spatial domain or in some transform domain (DCT, DFT, DWT) in a multiplicative manner:

$$
m_{n}=j_{n}+\gamma j_{n} y_{n}
$$

where $m_{n}$ is the $n^{t h}$ pixel of the watermarked image $M, j_{n}$ is the $n^{\text {th }}$ pixel of the cover image $J, y_{n}$ is the $n^{t h}$ pixel of the watermark $Y$ and $\gamma$ is known as the embedding factor and controls the watermark strength. The 

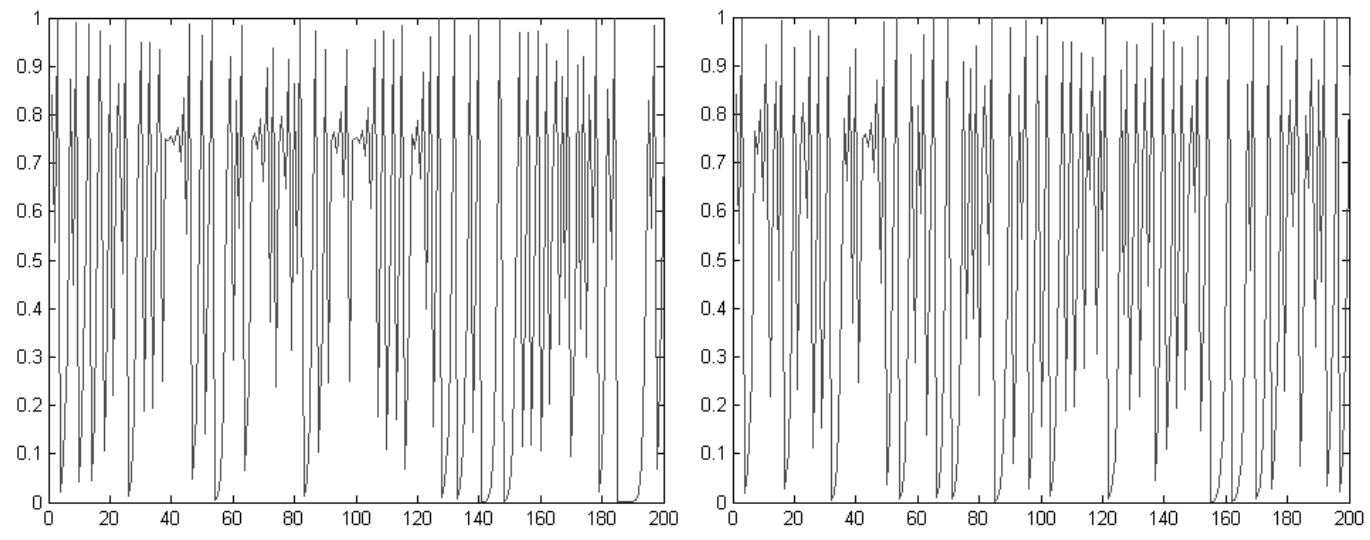

Figure 4. (a) Trajectory for logistic map with an initial value of 0.3 (b) Trajectory for logistic map with an initial value of 0.301 . Both cases are with $a=4$.

multiplicative watermark embedding rule relies on "Weber's Law", which indicates that the change in a stimulus that will be just noticeable is a constant ratio of the original stimulus, ${ }^{15}$ and may be expressed as:

$$
\frac{\Delta I}{I}=k
$$

where $I$ represents the initial intensity of a pixel, $\Delta I$ the change in the intensity of the pixel. $k$ signifies that the proportion on the left hand side remains constant despite the value of $I$ changing. In Eq. $6, \gamma$ controls the trade-off between watermark visibility and watermark robustness within the image. The lower the value of $\gamma$ the less noticeable a watermark is within an image and therefore does not noticeably alter the perceived quality of the image ${ }^{16}$.

The motivation for the use of an optical detection technique is that optical processing is faster than a similar digital technique due to the inherent parallelism of the system ${ }^{17}$. The optical correlation technique used is based on the Matched Filtering technique ${ }^{18,19}$ which has been shown to be an effective technique for watermark detection. For the optical detection system, the possibly watermarked image together with the watermark whose presence(or absence) in the image one wishes to determine are used as inputs to the detector. The watermark is used as the filter for the matched filter detector. The optical detection technique returns an output image which is the correlation between the possibly watermarked image and the watermark. For more detail on the optical detection scheme employed one may consult earlier work by the authors ${ }^{7,8}$.

\section{NON CHAOTIC REGIONS OF LOGISTIC MAP}

There are certain regions of the logistic map where chaotic behaviour is not observed. It should be obvious that no chaotic behaviour is observed for values of the seed $a<3.5699$. This point in the function is known as the accumulation point. Beyond this point the periodicity witnessed before it gives way to chaos. Within this chaotic region $(3.5699 \leq a \leq 4)$ there are non chaotic regions present. These occur at the bifurcation zones of the chaotic region of the map, for example, sizeable regions exist when $3.627 \leq a \leq 3.634$ ( 6 bifurcations), $3.739 \leq a \leq 3.744$ (5 bifurcations) and $3.829 \leq a \leq 3.856$ (3 bifurcations).

The latter of these regions, is known as a period-tripling region, and within this region the logistic map can be in one of three states. Using a seed for the function within this zone will lead to the system taking on only three values. The sequences produced are non chaotic, and also predictable and therefore are not suitable for watermark generation. A sample of a watermark generated when $a=3.83$ is shown in Fig. 5(a). In this case Peano Scanning is used to determine the scanning order of the pixels. Fig. 5(b) illustrates the watermark generated when $a=3.83$ and Raster Scanning is used. These figures illustrate that non-chaotic predictable sequences are produced even in the chaotic region. It can be seen that there is a pattern developing within the 
watermark and this is due to the predictability of the map at this location. Similar results are found for each of the non-chaotic regions of the map, indicated previously.
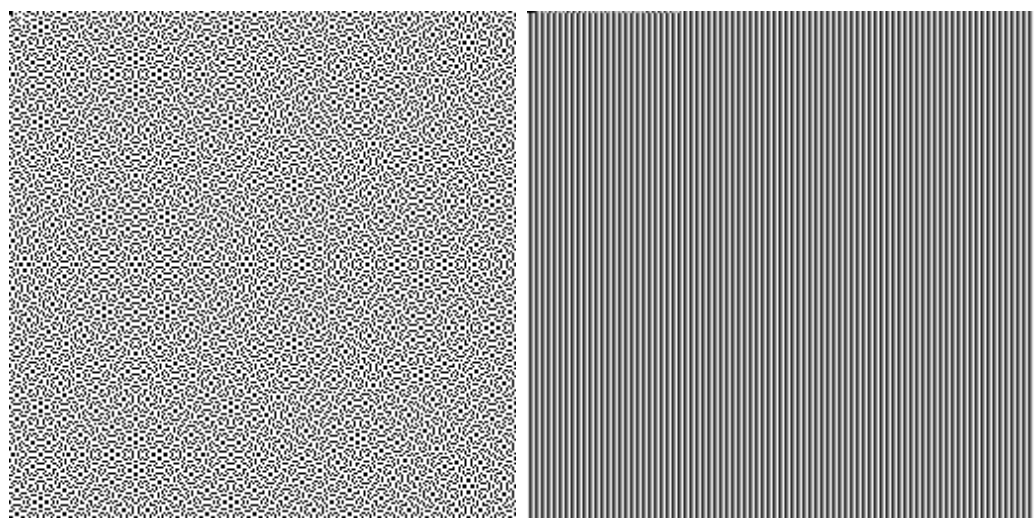

Figure 5. Watermark generated when (a) $a=3.83$ and incorporating Peano Scanning and (b) $a=3.83$ not incorporating Peano Scanning.

\section{PROBABILITY DENSITY}

It is useful to examine the frequency with which each state along a trajectory falls into a certain region of the state space and is known as the probability density or the invariant measure. and is calculated as follows: Assume that state space $[0,1]$ is divided into $n$ discrete nonintersecting intervals, so the $i^{\text {th }}$ interval is (neglecting the end point 1):

$$
[(i-1) / n, i / n) \quad i=1, \ldots, n
$$

Next an initial state $x_{0}$ is chosen and a trajectory of length $N(N>>n)$ calculated by:

$$
y_{0}, F\left(y_{0}\right), F^{2}\left(y_{0}\right)=F\left(F\left(y_{0}\right)\right), \ldots, F^{N}\left(y_{0}\right)
$$

Then it is straightforward to determine the fraction, $f_{i}$, of the $N$ system states that is in the $i^{t h}$ interval from ${ }^{20}$

$$
f_{i}=\frac{n}{N}\left\{\text { number of } \mathrm{F}^{\mathrm{j}}\left(\mathrm{y}^{0}\right) \in[(\mathrm{i}-1) / \mathrm{n}, \mathrm{i} / \mathrm{n}), \mathrm{j}=1, \ldots, \mathrm{N}\right\}
$$

Fig. 6 shows the calculated probability density when $a=4$ and when $a=3.83$ for 5000 iterations, and 200 possible states. It can be seen that in the case where $a=4$ there is symmetry with most states concentrated near 0 , and near 1 with a minimum at $\frac{1}{2}$. In the case of $a=3.83$ it can be seen that the system can be in only one of three possible states. This is not surprising since when $a=3.83$ one is within a stable state, the period tripling state, referred to earlier.

Fig. 7 shows the cases where the above procedure was repeated with $a=3.7$ and with $a=3.99$. It can be observed that in the case of $a=3.99$ there is a greater spread of states. There is a balance between the number of 0 pixels (white) and 1 pixels (black). In the case of $a=3.7$ it can be seen that the trajectory ends up in states greater than 0.5 substantially more times than in states less than 0.5 . This means that in the watermark there will be more 1 pixels than 0 pixels, i.e. more white than black pixels. In the case of $a=3.99$ there is much less density variation in watermark produced than in the case of the watermark generated when $a=3.7$. This suggests that the watermark produced when $a=3.99$ is more lowpass than the watermark produced when $a=3.7$ and has shown to be the case. ${ }^{8}$

There are however, other stable states within this region which are not suitable for usage in watermark generation. These stable states occur when a trajectory arrives at a fixed point of the logistic map. A fixed point 

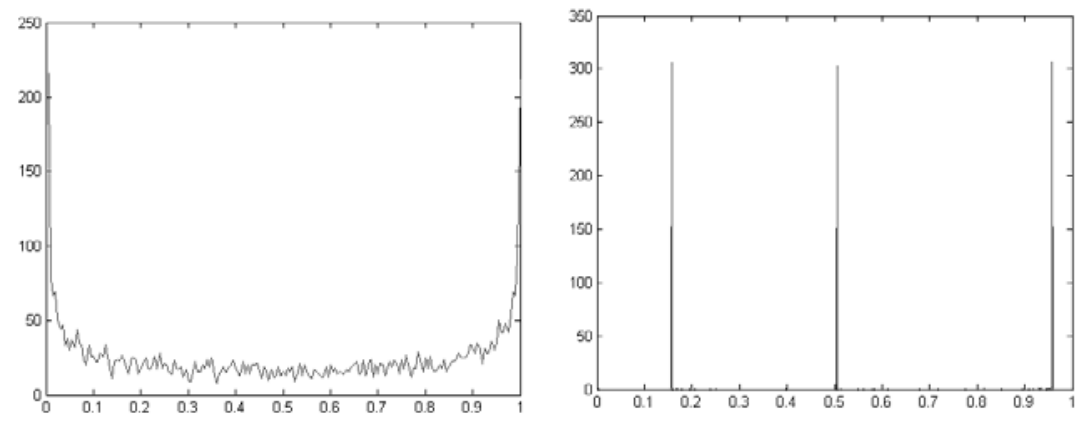

Figure 6. Probability Density when (a) $a=4$ and (b) $a=3.83$.
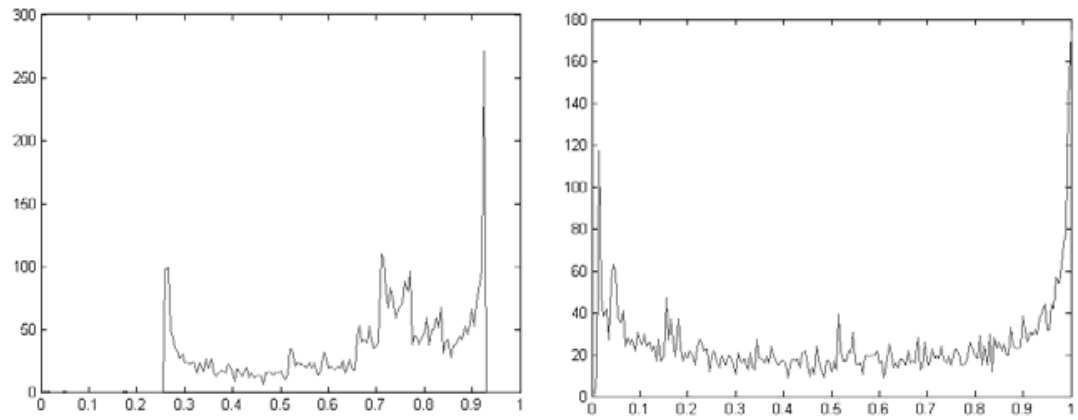

Figure 7. Probability Density when (a) $a=3.7$ and (b) $a=3.99$.

occurs whenever $y_{n+1}=y_{n}$. Solving this equation (and dropping the subscript) will give the values of these fixed points.

$$
\begin{aligned}
y[1-a(1-y)] & =y(1-a+a y) \\
& =a y\left[y-\left(1-a^{-1}\right)\right]=0
\end{aligned}
$$

Therefore the fixed points are

$$
y_{0}=0 \quad \text { and } \quad y_{0}=1-\mathrm{a}^{-1}
$$

and for the case of $a=4$ the two fixed points which satisfy Eq. 12 are $y_{0}=0$ and $y_{0}=\frac{3}{4}{ }^{20}$

\section{POWER SPECTRUM}

Pitas et al. advocate the use of the power spectrum to determine if a particular generated watermark sequence is lowpass or highpass in characteristics ${ }^{2}$. The power spectrum of a function is defined as the modulus-squared of the discrete Fourier Transform of some finite sampled section of it and is given by

$$
P(u, v)=|F T[I(u, v)]|^{2}
$$

where $P(u, v)$ is the power of a pixel $(u, v), F T$ is the Fourier Transform and $I(x, y)$ is the image pixel whose power spectrum one is calculating. For the logistic equation, the power spectrum is

$$
P\left(y_{n}\right)=\left|F T\left[y_{n}\right]\right|^{2}
$$

Examination of the power spectrum of the logistic map in the chaotic region, as shown in Fig. 8 shows that the general trend is a decrease in the power spectrum with increasing seed values. This suggests that more lowpass watermarks are generated with higher values of the seed $a$. However, we have observed that the power 


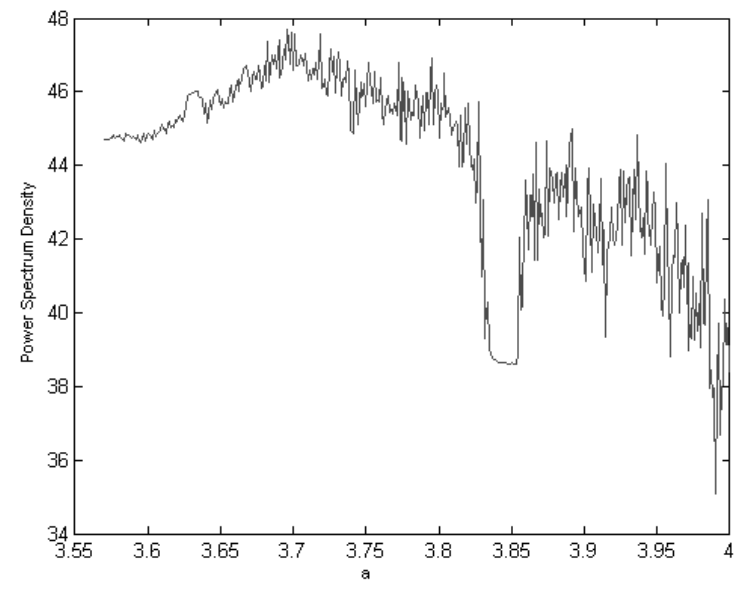

Figure 8. Power Spectrum Density for the chaotic region of the logistic map.

spectrum alone is not an indication of lowpass or highpass characteristics in the watermark. For example, it can be seen that either side of the period tripling region, $3.83 \leq a \leq 3.856$, there exists similar values of the power spectrum. One might expect that at these values of $a$ similar watermarks would be produced (for example, when $a=3.828$ and when $a=3.859$ ). It was found, however, that the watermark produced when $a=3.859$ was much more lowpass that the watermark produced when $a=3.828$ even though the power spectra were very similar. The generated watermarks are shown in Fig. 9 and the difference in the density variations in the watermark pixels can be easily observed.
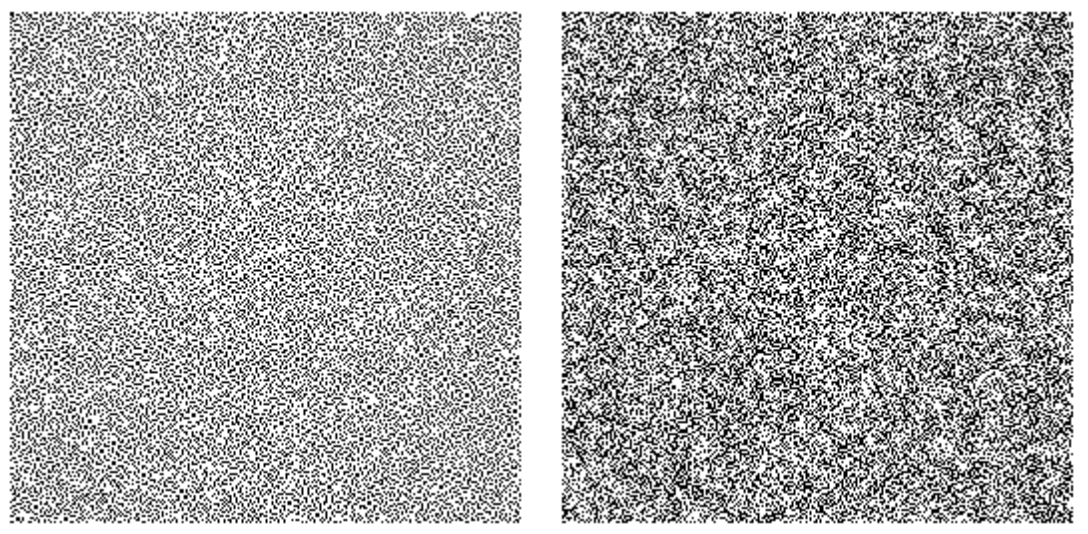

Figure 9. Watermark produced when (a) $a=3.828$ and (b) $a=3.859$.

A change in the spectral characteristics of the map occurs in this stable period tripling state. This is not the case with more well-defined chaotic functions like the Markov Map as the power spectrum is a smoother spectrum than that observed for the logistic map.

The watermarks generated using the logistic function have also been "attacked" using JPEG compression. JPEG is a lossy compression technique designed to exploit known limitations of the Human Visual System (HVS), notably the fact that small changes in the image will go unnoticed when viewed by a human. It was observed that in general as the watermarks used had more lowpass characteristics increased robustness to JPEG compression was observed. This is what is expected as lowpass watermarks have increased robustness with respect 
to image distortions that have lowpass characteristics (filtering, nonlinear filtering such as median filtering, lossy compression etc.). ${ }^{21}$

\section{RECOMMENDATIONS AND CONCLUSION}

In this section recommendations on using the logistic equation for watermark generation are provided. These suggestions are for chaos-based optically detectable watermarks which can be detected using an optical correlator. When deciding on a function seed for the logistic equation the user should be fully aware of the chaotic region of the map produced. They should be aware that although the section of the map from $3.57-4$ is commonly referred to as the chaotic region there are areas of this region that should be avoided. These occur when a bifurcation occurs in the map and it was presented that zones with 3,5 and 6 bifurcations existed.

Fixed states of a trajectory should also be avoided when selecting the function seed. If one of these fixed states is used to generate a watermark, there is no density variation (i.e. either a completely black or white watermark) which renders it unsuitable. There are two fixed points for each value of the function seed and they are known to occur when the initial value $y_{i}=0$ and $y_{i}=1-a^{-1}$. An example of a fixed state occurs when $a=4$ and with an initial value of $x_{0}=0$ or $x_{0}=\frac{3}{4}$.

Examination of the power spectrum shows that in general as the function seed increases more lowpass watermarks are generated. It has been shown that when noise is present in the optical detector a lowpass watermarks gives better performance ${ }^{8}$. It was also found that chaos-based watermarks with lowpass characteristics were more robust to JPEG compression. However, one can not rely on the power spectrum alone to determine whether a watermark is lowpass or otherwise. Reliance on the power spectrum for more ill-defined maps like the Logistic Map in determining relative lowpass properties is not recommended. 


\section{REFERENCES}

1. I. J. Cox, J. Killian, T. Leighton, and T. Shamoon, "Secure spread spectrum watermarking for images, audio, and video," Proceedings of International Conference on Image Processing (ICIP'96) III, pp. 243-246, 1996.

2. A. Tefas, A. Nikolaidis, N. Nikolaidis, V. Solachidis, S. Tsekeridou, and I.Pitas, "Markov chaotic sequences for correlation based watermarking schemes," Proceedings of Chaos, Solitons and Fractals 17, pp. 567-573, 2003.

3. A. Nikolaidis and I. Pitas, "Comparison of different chaotic maps with application to image watermarking," Proceedings of IEEE International Symposium on Circuits and Systems, Geneva, pp. 509-512, 2002.

4. S. Tsekeridou, V.Solachidis, N.Nikolaidis, A.Nikolaidis, A. Tefas, and I.Pitas, "Bernoulli shift generated watermarks: Theoretic investigation," Proceedings of IEEE Int. Conf. on Acoustics, Speech and Signal Processing, pp. 1989-1992, 2001.

5. Q. B. Sun, P. R. Feng, and R. Deng, "An optical watermarking solution for authenticating printed documents," Proceedings of International. Conference on Information Technology: Coding and Computing , pp. 65-70, 2001.

6. A. Herrigel, S. Voloshynovskiy, and Z. Hrytskiv, "An optical/digital identification/verification system based on digital watermarking technology," Proceedings of SPIE International Workshop on Optoelectronic and Hybrid Optical/Digital Systems for Image/Signal Processing, 1999.

7. A. Mooney and J. G. Keating, "Optical and digital technique for watermark detection," Proceedings of SPIE, Optical Information Systems 5202, pp. 97-105, 2003.

8. A. Mooney and J. G. Keating, "Noisy optical detection of chaos-based watermarks," SPIE Photonics North, Canada, 2004.

9. R. L. Devaney, A first course in Chaotic Dynamical Systems - Theory and Experiment, Perseus Books, Cambridge, Massachusetts, 1992.

10. M. Marek and I. Schreiber, Chaotic Behaviour of Deterministic Dissipative, Cambridge University Press, Cambridge, 1991.

11. M. R. Titchener and W. Ebeling, "Deterministic chaos and information theory," Procs. Data Compression Conference, 2001.

12. W. Ebeling, R. Steuer, and M. R. Titchener, "Partition-based entropies of deterministic and stochastic maps," Stochastics and Dynamics 1, 2001.

13. K. Yang and M. Mills, "Fractal based image coding scheme using peano scan," Proceedings of ISCAS ' 88 1470, pp. 2301-2304, 1988.

14. R. Stevens, A. F. Lehar, and F. Preston, "Manipulation and presentation of multidimensional image data using the peano scan," Proceedings of IEEE Trans. Patterm Pattern Anal. Machine Intell, pp. 520-526, 1983.

15. Encyclopaedia Brittanica Online, http://www.britannica.com/.

16. I. J. Cox, M. L. Miller, and J. A. Bloom, Digital Watermarking, Morgan Kaufmann, London, 2002.

17. J. Rosen, "Three-dimensional optical fourier transform and correlation," Optics Letters 22, pp. 964-966, 1997.

18. M. Shen, X. Zhang, L. Sun, P. J. Beadle, and F. H. Y. Chan, "A method for digital image watermarking using ica," 4th International Symposium on Independent Component Analysis and Blind Signal Separation , pp. 209-214, 2003.

19. A. Sequeira and D. Kundur, "Communication and information theory in watermarking: A survey," Proceedings of Multimedia Systems and Applications IV, A. G. Tescher, B. Vasudev, and V. M. Bove, eds., Proc. SPIE 4518, pp. 216 - 227, 2001.

20. A. Lasota and M. C. Mackey, Chaos, Fractals and Noise - Stochastic Aspects of Dynmaics, Springer, London, 1994.

21. J. Fridrich, "Combining low-frequency and spread spectrum watermarking," Proc. SPIE Int. Symposium on Optical Science, Engineering and Instrumentation, pp. 2-12, 1998. 